\title{
Thrombospondin in Renal Disease
}

\author{
Christian Hugo Christoph Daniel
}

Department of Nephrology and Hypertension, University Erlangen-Nürnberg, Erlangen, Germany

\section{Key Words}

Thrombospondin · Angiogenesis inhibitor

\begin{abstract}
Thrombospondins (TSPs) are multifunctional matricellular glycoproteins which are involved in the regulation of angiogenesis, proliferation, apoptosis, the NO-cGMP-dependent protein kinase pathway and transforming growth factor (TGF) $\beta$ activation. The TSP family consists of 5 members, but currently only data on effects of TSP- 1 and TSP- 2 in renal disease are available. Both TSPs are hardly expressed within the healthy renal cortex and can be upregulated during renal disease. Using different animal models for renal disease, TSP1 and -2 were found to be important regulators of pathophysiological changes during renal disease with similar and contrary effects. TSP-1 is a major activator for TGF- $\beta$ resulting in profibrotic effects in the injured kidney. In contrast, TSP-2 lacks the ability for its activation. Proapoptotic actions of TSP-1 were found during renal ischemia/reperfusion injury. While TSP-1 exerts proinflammatory actions, the currently available data for TSP-2 propose anti-inflammatory effects for this molecule. Both TSPs are known angiogenesis inhibitors, which could be proved for TSP-2, but antiangiogenic effects for TSP-1 were only evident by treatment with TSP-1 peptides in renal disease. In addition, TSP-2 can inhibit cell proliferation and matrix metalloproteinase 2 activity.
\end{abstract}

Copyright $\odot 2009$ S. Karger AG, Basel (c) 2009 S. Karger AG, Basel

$1660-2129 / 09 / 1113-0061 \$ 26.00 / 0$

Accessible online at:

www.karger.com/nee

\section{Introduction}

Thrombospondin 1 (TSP-1) is a multifunctional homeotrimeric matricellular glycoprotein of $450 \mathrm{kDa}$ and is expressed by a variety of cell types such as platelets (high amounts), vascular smooth muscle cells as well as diverse renal cells including mesangial cells, podocytes, endothelial cells, tubular and interstitial cells. TSP-1 is the founding member of a small family of 5 secreted, matricellular proteins (TSP-1 to TSP-5), whereas TSP-2 is most similar to TSP-1, both of which are trimeric, while TSP-3 to TSP-5 belong to a second subclass of pentameric proteins.

During embryonic development, TSP-1 and TSP-2 are expressed in various tissues with overlapping and exclusive localizations. In healthy adults, TSP-1 more than TSP-2 is regulated by various cytokines such as plateletderived growth factor, fibroblast growth factor 2 or transforming growth factor (TGF) $\beta$, and is frequently expressed at sites of inflammation, wound healing and tissue remodeling [1].

Loss of TSP-1 or TSP-2 expression in null mice produces relatively normal mice with minor defects in skeletal development, fertility and lung homeostasis (TSP-1) or connective tissue remodeling (TSP-2) [1,2]. In vitro studies in various cell types have demonstrated multiple functions of TSP-1/2 in modulating platelet aggregation, cell proliferation, survival, adhesion and migration, strongly depending on its interacting partners [1]. Hereby, TSP-1/2 bind many growth factors, proteases, cell sur-

Christian Hugo, MD

Department of Nephrology and Hypertension, Universität Erlangen-Nürnberg Krankenhausstrasse 12, DE-91054 Erlangen (Germany)

Tel. +49913185 39002, Fax +4991318539209

E-Mail Christian.Hugo@rzmail.uni-erlangen.de 
face receptors and extracellular matrix proteins mediating specific cellular responses including the regulation of TGF- $\beta$ activity. The various biological activities of TSP-1 relate to specific domains of the complex molecule including an aminoterminal heparan binding region, a procollagen-like region with intermolecular disulfide bonds, 3 properdin-like type 1 repeats, 3 epidermalgrowth-factor-like type 2 repeats, a calcium-sensitive type 3 repeat and a C-terminal globular domain. Multiple receptors such as heparan sulfate, calreticulin, lipoprotein-receptor-related protein, CD $36, \alpha v \beta 3$-integrin, CD47 as well as specific peptide sequences such as the TGF- $\beta$-activating sequence RFK have been identified to be responsible for certain actions. Typically, these TSP-1 or TSP-2 null mice need to be exposed to specific stresses/ injuries to reveal their phenotypes.

In this article, the possible involvement of TSP-1/2 in experimental renal disease is discussed, while almost no information is available regarding expression and role of TSP-3 to TSP-5 in kidney disease.

\section{TSP in Glomerulonephritis}

In the normal, healthy renal cortex, TSP-1 is hardly expressed. During human and experimental glomerulonephritis characterized by inflammatory changes in glomerular capillaries with cellular injury and progressive extracellular matrix accumulation, TSP-1 is transiently highly expressed at sites of injury and inflammation by a large variety of endogenous renal and inflammatory cell types.

Lack or blockade of TSP-1 is in general beneficial for the time course of experimental glomerulonephritis as indicated by several studies in rodents.

Hochegger et al. [3] compared the time course of TSP1 null with wild-type mice in the autologous phase of an accelerated model of anti-glomerular-basement-membrane (GBM) nephritis. Twenty-one days after disease induction, TSP-1 null mice demonstrated decreased proteinuria, serum creatinine levels as well as improved renal histology. Hereby, histological disease indices such as crescent formation, fibrin deposition, influx of inflammatory cells such as macrophages and $\mathrm{T}$ lymphocytes were reduced in TSP-1 null versus wild-type mice suggesting a proinflammatory effect of TSP-1 in this renal disease model. The specific mechanism of how TSP-1 mediates these effects remains unclear.

Our group investigated the role of TSP-1 in a rat model of complement-mediated mesangial proliferative glo- merulonephritis induced via injection of an antibody against the Thyl antigen on mesangial cells, the antiThyl model. This model mimics typical features of human mesangial proliferative glomerulonephritis, the most common glomerulonephritis in the western world. In this model, TSP-1 as well as its potential interacting partner protein TGF- $\beta$ are transiently upregulated during disease. The role of TGF- $\beta$ as a major profibrotic cytokine in various fibrotic diseases in multiple organ systems as well as in the anti-Thyl model has been well established [4]. While these studies suggest great benefit from suppression of TGF- $\beta$ function in fibrotic kidney disease, TGF- $\beta_{1}$ null mice die a few weeks after birth from a severe generalized inflammatory response demonstrating that complete suppression of TGF- $\beta$ function must not be a therapeutic goal in treating inflammatory kidney disease [5]. Therefore, accurate regulation of TGF$\beta$ function seems to be critical for the health of mammals, and any anti-TGF- $\beta_{1}$ therapeutic approach should try to target the local overproduction (and function) of TGF- $\beta$ as specifically as possible. One possibility to approach this goal could be by controlling the activation process of locally produced TGF- $\beta$.

\section{TSP and TGF- $\beta$ Activation}

TGF- $\beta$ is secreted by most cell types as a latent, inactive procytokine complex that consists of the mature, active TGF- $\beta$ protein, which is noncovalently bound to a dimer of its N-terminal propeptide, the so-called latencyassociated protein (LAP), and variably to a latent TGF- $\beta$ binding protein [5]. The mature TGF- $\beta$ protein has to be extracellularly released from this procytokine complex to be able to interact with its receptors. Among various players/mechanisms such as $\mathrm{pH}$ changes, gamma irradiation, reactive oxygen species, plasmin, calpain or cathepsin, recent data have suggested TSP-1 as an activator of TGF- $\beta_{1}$ in vitro in different cell systems as well as in cellfree systems [6, 7] (fig. 1). It has been demonstrated that TSP-1 forms a trimolecular complex with the TGF- $\beta$ procytokine complex by interacting with the mature TGF- $\beta$ protein as well as the LAP. Hereby, the hexapeptide GGWSXW from the type 1 repeat of the TSP-1 molecule is required for TSP- 1 binding to the mature TGF- $\beta$ protein via its VLAL site [8]. This now allows the molecular interaction of the KRFK amino acid sequence of the TSP1 molecule with the N-terminal LSKL sequence of the LAP $[7,9]$. It is the RKPK sequence of the mature TGF- $\beta$ that is required for binding to the LSKL sequence of the 
LAP [8]. TSP-1, KRFK peptides, but also RKPK peptides have been shown to activate latent TGF- $\beta$, presumably by disrupting interactions between LAP and mature TGF- $\beta$, while TSP-2 (binding also to the mature TGF- $\beta$ protein via its GGWSXW sequence but lacking the KRFK region for activation) and both, the hexapeptide GGWSXW and the LSKL peptide, are able to block activation of TGF- $\beta$ by TSP-1 $[7,9]$.

To evaluate whether TSP-1 is an endogenous activator of TGF- $\beta$ in vivo in the anti-Thyl model, we demonstrated specific inhibition of TGF- $\beta$ activation using two independent methods targeting interactions of TSP-1 or TSP-1 with TGF- $\beta$ in vivo, first by renal arterial transfer of antisense phosphorothioate oligodeoxynucleotide against TSP-1 into glomeruli and second by continuous intravenous infusion of specific peptides interfering with the activation of TGF- $\beta$ by TSP-1 (GGWSXW or LSKL as indicated above) compared to appropriate controls $[10$, 11]. In these studies, successfully treated rats showed a decrease in glomerular TGF- $\beta$ activity and/or TGF- $\beta$ receptor signaling as well as glomerular extracellular matrix accumulation as assessed by trichrome, collagen I, $\mathrm{IV}$, and fibronectin staining and proteinuria, but no change regarding glomerular cell proliferation, cell death or macrophage accumulation compared to control rats. These were the first studies demonstrating that TSP-1 is an endogenous activator of TGF- $\beta$ but did not act as an endogenous angiogenesis inhibitor (no effect on endothelial proliferation in both experiments) in vivo in experimental renal disease. In contrast, therapeutic treatment with peptides derived from the antiangiogenic sequence of the TSP-1 molecule decreased glomerular endothelial and mesangial cell proliferation as well as proteinuria in the anti-Thyl model [12].

\section{TSP-2 in Renal Inflammation and Angiogenesis}

In contrast to TSP-1, TSP-2 could be identified as an endogenous antiangiogenic, but not as a TGF- $\beta$-activityregulating factor in renal disease, comparing TSP-2 null with wild-type mice in an anti-GBM nephritis model [13]. Similar to TSP-1, TSP-2 protein was hardly detectable within the renal cortex of normal control wild-type mice but was clearly upregulated during anti-GBM disease. Contrary to TSP-1, TSP-2 deficiency led to an accelerated and enhanced inflammatory response, as indicated by the influx of $\mathrm{T}$ lymphocytes and monocytes/macrophages, as well as glomerular fibrin deposition and a matrix-remodeling response as indicated by collagen I

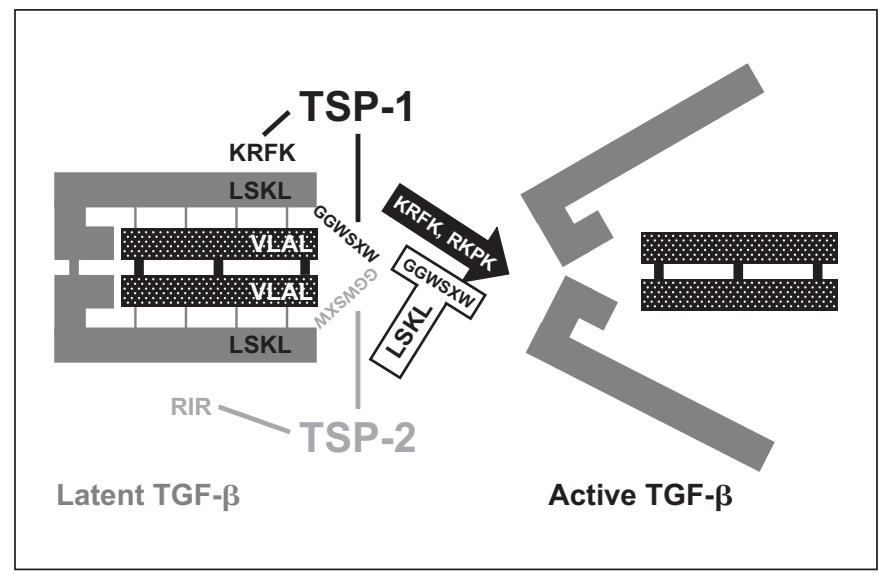

Fig. 1. TSP-1-mediated TGF- $\beta$ activation. The hexapeptide GGWSXW from the type 1 repeat of the TSP-1 molecule (black) is required for TSP-1 binding to the mature TGF- $\beta$ protein via its VLAL site (dotted black). This now allows the molecular interaction of the KRFK amino acid sequence of the TSP-1 (black) molecule with the N-terminal LSKL sequence of the LAP (gray). TSP1, KRFK peptides but also RKPK peptides have been shown to activate latent TGF- $\beta$, presumably by disrupting interactions between LAP and mature TGF- $\beta$ (black arrow), while TSP-2 (light gray, binding also to the mature TGF- $\beta$ protein via its GGWSXW sequence but lacking the KRFK region for activation) and both the hexapeptide GGWSXW and the LSKL peptide are able to block activation of TGF- $\beta$ by TSP-1 (white blocking bar).

and IV staining, and by the proliferative response within the renal interstitium. The markedly accelerated and enhanced proliferative response of the renal peritubular endothelium led to improved reversal of capillary rarefaction in TSP-2 null mice, while interstitial cell death was equivalent to wild-type mice. These changes were accompanied by increased matrix metalloproteinase 2 activity but not by increased TGF- $\beta$ activity in the TSP- 2 null mice.

\section{TSP-1 in Diabetic Nephropathy}

Diabetic nephropathy is the most common disease leading to renal replacement therapy in the western world and is also associated with increased production and activity of TGF- $\beta$ [5]. Experimental studies showed that many features of diabetes nephropathy such as hypertrophy and hyperfiltration, matrix accumulation, GBM thickening and podocyte loss are dependent on TGF- $\beta$ [14]. In vitro studies using cultured mesangial cells demonstrated that under high glucose conditions, TGF- $\beta$ ac- 
tivation is mediated via TSP-1 [15]. Hereby, TSP-1 transcription is stimulated by glucose via TSP-1 promoter binding of the nuclear protein upstream stimulatory factor 2 (USF2). Glucose-mediated USF2 accumulation is dependent on protein kinase C, p38 mitogen-activated protein kinase and extracellular signal-regulated kinase pathways. Increased NO-cGMP-dependent protein kinase activity is able to downregulate USF2 protein levels and its DNA binding activity, resulting in decreased TSP1 transcription and TGF- $\beta$ activity in cultured rat mesangial cells [16]. Overexpression of USF2 in cultured rat mesangial cells reverses the inhibitory effect of cGMPdependent protein kinase on glucose-induced TSP-1 transcription and TGF- $\beta$ activity.

These in vitro results are complemented by in vivo experiments using USF2 transgenic mice, which were exposed to streptozotocin-induced diabetes mellitus [17]. Overexpression of USF2 in transgenic mice accelerated several features of streptozotocin-induced diabetic nephropathy including hypertrophy, matrix accumulation and proteinuria compared to wild-type diabetic mice 15 weeks after disease induction. Hereby, USF2 transgenic mice exhibited increased USF2 expression, DNA binding activity, TSP-1 mRNA and protein levels, as well as TGF$\beta$ activity in diabetic glomeruli.

In contrast, in TSP-1 null compared to wild-type mice, the time course of streptozotocin-induced diabetic nephropathy is attenuated as demonstrated by a significant reduction of glomerulosclerosis, glomerular matrix accumulation, podocyte injury, renal infiltration with inflammatory cells as well as renal functional parameters [18]. In diabetic TSP-1 null mice, the amount of active TGF- $\beta$ within glomeruli was significantly lower as indicated by staining with specific antibodies either against active TGF- $\beta$ or the TGF- $\beta$-signaling molecule phospho$\operatorname{smad} 2 / 3$ or the typical TGF- $\beta$ target gene product plasminogen activator inhibitor 1 , while the amount of glomerular total TGF- $\beta$ remained unchanged. These results demonstrate that TSP-1 is an important activator of TGF$\beta$ also in diabetic nephropathy in vivo.

These experimental results may also be relevant for a potentially therapeutic strategy in human diabetic nephropathy, since TSP-1 is upregulated in kidney biopsies from patients with diabetic nephropathy and correlates spatially with TGF- $\beta$ expression and TGF- $\beta$ receptor signalling as indicated by phosphorylation of the signal transduction molecule smad2/3 [19]. In addition, Moczulski et al. [20] investigated the gene expression profile of blood mononuclear cells from type 1 diabetic patients with and without diabetic nephropathy using the Af- fymetrix microarray system. Hereby, out of 198 candidate genes (according to a Pubmed search), which were suspected of being involved in the pathogenesis of diabetic nephropathy, only cyclooxygenase 1 and TSP-1 were overexpressed in mononuclear cells from patients with diabetic nephropathy versus those without nephropathy.

\section{TSP-1 in Acute Renal Failure}

While high-glucose-mediated TSP-1 expression and TGF- $\beta$ activation can be repressed via activation of the $\mathrm{NO}$-cGMP-dependent protein kinase pathway, recent data demonstrate TSP-1 also as a physiological, potent antagonist of the vasoprotective and proangiogenic NOsoluble guanylate cyclase-cGMP pathway in platelets, vascular smooth muscle cells and endothelial cells [21]. Hereby, TSP-1 controls multiple steps in the NO-soluble guanylate cyclase-cGMP signaling cascade by acting primarily through binding to its cell receptor CD47 to limit soluble guanylate cyclase and cGMP-dependent protein kinase activation. In addition, TSP-1 signals through its receptor $\mathrm{CD} 36$ to inhibit this pathway by controlling the activation of the nitric oxide synthase isoform NOS3. This NO-cGMP antagonistic role of TSP-1 seems to be especially relevant for tissue survival in the situation of tissue ischemia. Animal studies demonstrated that endogenous TSP-1, via its receptor CD47, limits blood flow, vascular remodeling and tissue survival in both a fixed ischemic injury and skin grafts [21].

Similarly, TSP-1 was found to affect tissue survival in an acute renal failure model in mice. Using an ischemia/ reperfusion model, Thakar et al. [22] found TSP-1 highly upregulated early after induction of injury ( $3 \mathrm{~h}$ ) in damaged tubuli. Colocalization studies with activated caspase 3, as a marker for apoptosis, revealed that TSP-1 was predominantly expressed in cells undergoing programmed cell death. The proapoptotic effect of TSP-1 on proximal tubular cells was also supported by studies in vitro. In addition, the deleterious role of TSP-1 in ischemia/reperfusion injury was shown by comparison of kidney structure and function in wild-type mice with TSP-1 null mice [22]. Mice lacking TSP-1 showed significant protection against renal failure as demonstrated by reduced serum creatinine $24 \mathrm{~h}$ after model induction. Furthermore, tubular damage was reduced in injured TSP-1 null mice, as demonstrated by significant reduction in brush border loss, cell necrosis, tubular dilation, cast formation and vacuolization when compared with 
Fig. 2. Known functions of TSP- 1 and -2 in renal disease. The role of TSPs was investigated in different animal models for renal diseases as indicated by superscript numbers. 1 = Anti-Thyl nephritis, a model for mesangioproliferative glomerulonephritis; 2 = streptozotocin-induced diabetes as a model for diabetic nephropathy; $3=$ anti-GBM nephritis model; $4=$ renal ischemia/reperfusion model. Renal antiangiogenic effects for TSP-1 were only found by pharmacological treatment with TSP-1 peptides (dotted box). MMP = Matrix metalloproteinase.

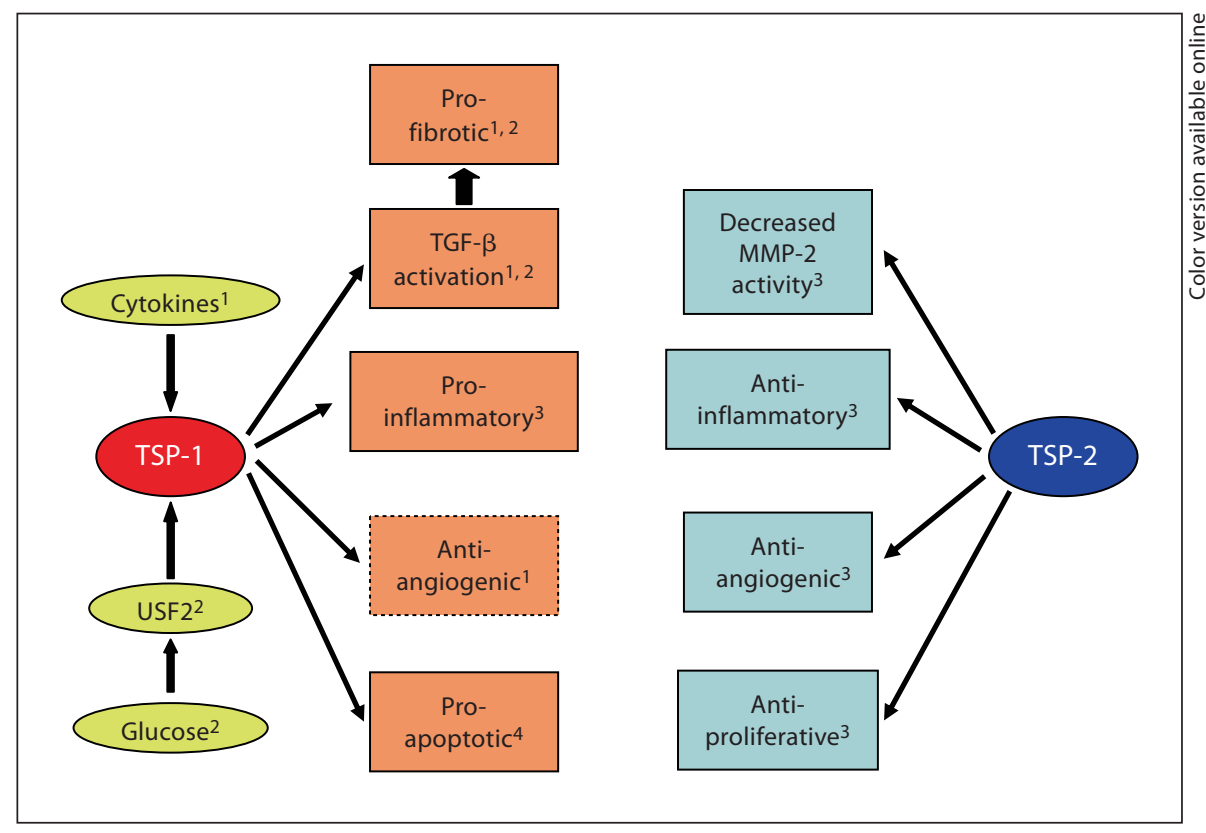

wild-type animals. The proapoptotic function of TSP-1 in ischemia/reperfusion injury was confirmed, since tubular apoptosis was significantly lower in the TSP-1 null mice. Hereby, TSP-1 is an important mediator of injury in kidney ischemia.

\section{Conclusion}

In summary, TSP-1 and -2 are important regulators of pathophysiological changes during renal disease with similar and contrary effects (fig. 2). TSP-1 is a major activator for TGF- $\beta$ resulting in profibrotic effects in the injured kidney. In contrast, TSP-2 can also bind the latent TGF- $\beta$ complex but lacks the ability for its activation.
Hereby, TSP-2 can potentially act as competitive inhibitor for TSP-1-mediated TGF- $\beta$ activation. Proapoptotic actions of TSP-1 were found during renal ischemia/reperfusion injury. While TSP-1 exerts proinflammatory actions, the currently available data for TSP-2 propose antiinflammatory effects for this molecule. Both TSPs are known angiogenesis inhibitors, which could be proved for TSP-2, but antiangiogenic effects for TSP-1 were only evident by treatment with TSP-1 peptides in renal disease. In addition, TSP-2 can inhibit cell proliferation and matrix metalloproteinase 2 activity.

Therefore, TSP-1 is a promising target for antifibrotic therapy in renal disease. Future experiments using gene therapy will clarify if TSP-2 can inhibit deleterious functions of TSP-1 in renal disease.

\section{References}

1 Bornstein P: Thrombospondins as matricellular modulators of cell function. J Clin Invest 2001;107:929-934.

2 Lawler J, Sunday M, Thibert V, Duquette M, George EL, Rayburn H, Hynes RO: Thrombospondin-1 is required for normal murine pulmonary homeostasis and its absence causes pneumonia. J Clin Invest 1998;101: 982-992.
Hochegger K, Knight S, Hugo C, Mayer G, Lawler J, Mayadas TN, Rosenkranz AR: Role of thrombospondin-1 in the autologous phase of an accelerated model of anti-glomerular basement membrane glomerulonephritis. Nephron Exp Nephrol 2004;96:e31e38.

4 Border WA: Transforming growth factorbeta and the pathogenesis of glomerular diseases. Curr Opin Nephrol Hypertens 1994;3: 54-58.
5 Bitzer M, Sterzel RB, Bottinger EP: Transforming growth factor-beta in renal disease. Kidney Blood Press Res 1998;21:1-12.

6 Lawrence DA: Transforming growth factorbeta: a general review. Eur Cytokine Netw 1996;7:363-374.

7 Ribeiro SM, Poczatek M, Schultz-Cherry S, Villain M, Murphy-Ullrich JE: The activation sequence of thrombospondin-1 interacts with the latency-associated peptide to regulate activation of latent transforming growth factor-beta. J Biol Chem 1999;274: 13586-13593. 
8 Young GD, Murphy-Ullrich JE: The tryptophan-rich motifs of the thrombospondin type 1 repeats bind vlal motifs in the latent transforming growth factor-beta complex. J Biol Chem 2004;279:47633-47642.

$>9$ Schultz-Cherry S, Murphy-Ullrich JE: Thrombospondin causes activation of latent transforming growth factor-beta secreted by endothelial cells by a novel mechanism. J Cell Biol 1993;122:923-932.

-10 Daniel C, Takabatake Y, Mizui M, Isaka Y, Kawashi H, Rupprecht H, Imai E, Hugo C: Antisense oligonucleotides against thrombospondin-1 inhibit activation of TGF-beta in fibrotic renal disease in the rat in vivo. Am J Pathol 2003; 163:1185-1192.

-11 Daniel C, Wiede J, Krutzsch HC, Ribeiro SM, Roberts DD, Murphy-Ullrich JE, Hugo C: Thrombospondin-1 is a major activator of TGF-beta in fibrotic renal disease in the rat in vivo. Kidney Int 2004;65:459-468.

- 12 Hugo CP, Pichler RP, Schulze-Lohoff E, Prols F, Adler S, Krutsch HC, Murphy-Ullrich JE, Couser WG, Roberts DD, Johnson RJ: Thrombospondin peptides are potent inhibitors of mesangial and glomerular endothelial cell proliferation in vitro and in vivo. Kidney Int 1999;55:2236-2249.
-13 Daniel C, Amann K, Hohenstein B, Bornstein P, Hugo C: Thrombospondin 2 functions as an endogenous regulator of angiogenesis and inflammation in experimental glomerulonephritis in mice. J Am Soc Nephrol 2007;18:788-798.

14 Ziyadeh FN: Mediators of diabetic renal disease: the case for TGF-beta as the major mediator. J Am Soc Nephrol 2004;15(suppl 1): S55-S57.

15 Poczatek MH, Hugo C, Darley-Usmar V, Murphy-Ullrich JE: Glucose stimulation of transforming growth factor-beta bioactivity in mesangial cells is mediated by thrombospondin-1. Am J Pathol 2000;157:1353-1363.

16 Wang S, Skorczewski J, Feng X, Mei L, Murphy-Ullrich JE: Glucose up-regulates thrombospondin 1 gene transcription and transforming growth factor-beta activity through antagonism of cGMP-dependent protein $\mathrm{ki}$ nase repression via upstream stimulatory factor 2. J Biol Chem 2004;279:3431134322 .

17 Liu S, Shi L, Wang S: Overexpression of upstream stimulatory factor 2 accelerates diabetic kidney injury. Am J Physiol Renal Physiol 2007;293:F1727-F1735.
18 Daniel C, Schaub K, Amann K, Lawler J, Hugo C: Thrombospondin-1 is an endogenous activator of TGF-beta in experimental diabetic nephropathy in vivo. Diabetes 2007; 56:2982-2989.

19 Hohenstein B, Daniel C, Hausknecht B, Boehmer K, Riess R, Amann KU, Hugo CP: Correlation of enhanced thrombospondin-1 expression, TGF- $\beta$ signalling and proteinuria in human type-2 diabetic nephropathy. Nephrol Dial Transplant 2008;23:38803887.

20 Moczulski DK, Fojcik H, Wielgorecki A, Trautsolt W, Gawlik B, Kosiorz-Gorczynska S, Oczko-Wojciechowska M, Wiench M, Strojek K, Zukowska-Szczechowska E, Grzeszczak W: Expression pattern of genes in peripheral blood mononuclear cells in diabetic nephropathy. Diabet Med 2007;24:266271.

21 Isenberg JS, Frazier WA, Roberts DD: Thrombospondin-1: a physiological regulator of nitric oxide signaling. Cell Mol Life Sci 2008;65:728-742.

22 Thakar CV, Zahedi K, Revelo MP, Wang Z, Burnham CE, Barone S, Bevans S, Lentsch $\mathrm{AB}, \mathrm{Rabb} \mathrm{H}$, Soleimani M: Identification of thrombospondin 1 (TSP-1) as a novel mediator of cell injury in kidney ischemia. J Clin Invest 2005;115:3451-3459. 